\title{
Suppressing stellar residual light on extremely large telescopes by aperture modulation
}

\author{
Szymon Gladysz ${ }^{1, a}$, Erez Ribak ${ }^{2}$, Bruno Femenia Castella ${ }^{3}$, Laurent Jolissaint ${ }^{4}$, Patrice Martinez ${ }^{1}$, \\ Remi Soummer ${ }^{5}$, Emmanuel Aller Carpentier ${ }^{1}$, Markus Kasper ${ }^{1}$, and Julian Christou ${ }^{6}$ \\ 1 European Southern Observatory, Karl-Schwarzschild-Strasse 2, 85748 Garching, Germany. \\ 2 Physics Department, Technion - Israel Institute of Technology, Haifa 32000, Israel \\ 3 Instituto de Astrofisica de Canarias, C/ Via Lactea, s/n, E38205 - La Laguna, Spain \\ 4 Leiden Observatory, University of Leiden, Niels Bohrweg 2, NL-2333 CA Leiden, The Netherlands \\ 5 Space Telescope Science Institute, 3700 San Martin Drive, Baltimore, MD 21218, USA \\ 6 Gemini Observatory, Hilo, Hawaii 96720, USA
}

\begin{abstract}
Efficient suppression of the residual stellar light is paramount to the success of exoplanet imaging efforts. We present a new approach to diffraction suppression on extremely large telescopes. The method is based on the realization that by obscuring part of the wavefront in the Lyot stop we can change the shape of the corresponding PSF. This obscuration is then modulated leading to modulated Airy pattern, while image of a planet - if present - is only slightly affected. The modulation could then be removed from the set of images, leading to better SNR of the possible planets. Simulation of the European Extremely Large Telescope has been used to test this approach.
\end{abstract}

\section{Introduction}

Imaging exoplanets requires extreme adaptive optics, coronagraphy and post-processing methods. The capability of a planet imager to detect faint companions will in the end be limited by its ability to remove stellar light. Extreme adaptive optics increases the spatial coherency of the wavefront, as quantified by the Strehl ratios above 90\% [1] [2]. Coronagraphy [3] [4] [5] removes this coherent part of the wavefront, thus decreasing residual stellar Poisson noise and increasing time to saturation of the detector. Coronagraphs also remove some of the speckle noise, in particular "pinned" speckles which result from amplification of intensity fluctuations by the deterministic Airy pattern [6] [7]. Post-processing methods [8] [9] [10], most of which involve PSF subtraction, can suppress some of the speckle noise contribution.

We propose a novel and simple technique to suppress the Airy pattern and speckle noise. By temporally modulating the shape of the Lyot stop after the focal-plane mask we distort the shape of the resulting coronagraphic PSF, shifting the Airy pattern and static speckles between pixels of the detector. The image of the planet stays in the same location. After a modulation sequence is completed the resulting multi-frame dataset can be analyzed in such a way as to remove the modulated stellar light, for example with a "dark speckle" algorithm [11]. This method is perfectly suited for ELTs which will produce saturated images of bright stars within seconds, and therefore multi-frame imaging will be a necessity.

The proposed method is a direct extension of aperture modulation ("symmetry breaking") [12]. In our previous work we used an off-centered mask to block part of the wavefront in the conjugate pupil plane. This mask was then rotated. At each rotation step of 9 degrees we recorded one image. The time sequence of intensity in each pixel of the image is modulated by the mask rotation. Only a very small part of this intensity stays constant, that of the planet, if it exists at a particular location. We

a e-mail: sgladysz@eso.org

This is an Open Access article distributed under the terms of the Creative Commons Attribution-Noncommercial License, which permits unrestricted use, distribution, and reproduction in any noncommercial medium, provided the original work is properly cited. 
found that by using a variant of the "dark speckle" method, basically displaying the minimum of intensity for each pixel, we could reveal the planets which were otherwise hidden in the long-exposure images taken with a static imaging system. In a monochromatic simulation of VLT/SPHERE planets $10^{5}$ fainter than the star, separated by $0.5 \operatorname{arcsec}(12.5 \lambda / \mathrm{D}$ in H-band) were easily detectable after an observation lasting $100 \mathrm{sec}$. Here we report on some improvements to the method and demonstrate its advantages over standard, "static" coronagraphy.

\section{Simulations}

We model a high-contrast imaging system on the $42 \mathrm{~m}$ European Extremely Large Telescope (E-ELT). The simulated adaptive optics (AO) system has parameters similar to the planned EPICS instrument [13], i.e. inter-actuator distance of $0.2 \mathrm{~m}$ (same as SPHERE), wavefront sensor (WFS) integration time of $1 \mathrm{~ms}$, AO loop lag of $1 \mathrm{~ms}$, and a WFS readout noise of $0.5 \mathrm{e}^{-}$. We model a Hartmann-Shack WFS as opposed to the EPICS baseline choice of a pyramid WFS. The simulation is monochromatic with the central wavelength of $2.2 \mu \mathrm{m}$ and a filter width of $0.4 \mu \mathrm{m}$.

The action of $\mathrm{AO}$ is modelled in an analytic fashion. For example the AO fitting error can be described as a result of the high-pass transfer function acting on the Kolmogorov atmospheric power spectrum of phase. The star of magnitude 7 is observed on-axis therefore we consider the transfer functions corresponding to the fitting error, the WFS noise error, and the AO loop delay error, but neglect the anisoplanatic transfer function. The WFS aliasing effect is also neglected because we assume a spatial filter in front of the WFS. The PAOLA simulation package [14] was used to model the action of $\mathrm{AO}$ on the atmospheric power spectrum. The modelled atmosphere consists of 10 layers. The heights, fractional strengths, and wind speeds in the layers were taken from the model which is being extensively used in simulations of the E-ELT, as part of the E-ELT Design Reference Mission [15].

The AO-filtered phase spectrum was used to generate independent realizations of AO residual phase using a classic, Fourier-domain filtering algorithm [16]. To this phase two static components have been added: static aberrations from the main mirror's segments (originally $50 \mathrm{~nm}$ total rms divided evenly between the piston, tip/tilt, coma, and astigmatism contributions; subsequently filtered by AO to give $15 \mathrm{~nm}$ rms of residual mirror aberrations reaching the coronagraph), and the non-common-path aberrations not sensed by $\mathrm{AO}$ and modelled with an $\mathrm{f}^{-2}$ spectrum [17] and the rms value of $30 \mathrm{~nm}$. The instantaneous PSFs corresponding to these wavefronts had Strehl ratios around 95\%.

The action of a coronagraph is simulated in the following way. The electric field is Fourier-transformed to give the amplitude PSF in the focal plane. The result is multiplied by the representation of the focalplane mask, taken to be $7.5 \lambda / \mathrm{D}$ in diameter. This value has been optimized for the inner working angle of $3.9 \lambda / \mathrm{D}$ and the current design of the E-ELT main mirror, i.e. $30 \%$ linear obscuration ratio [5]. The electric field after the focal-plane mask is inverse Fourier-transformed and multiplied by the model of the Lyot stop. For the Lyot coronagraph this Lyot stop is static and has an oversized central obscuration and undersized outer edge, resulting in the throughput of $67 \%$. In the second simulation this aperture-conjugate, round Lyot stop changes shape with time. The modulation consists of three contributions: blocking of part of the wavefront by moving the stop off-axis, rotation of the displaced stop, and decrease of its linear dimension with time. By inspecting the final images of star-planet systems we found that the optimal parameters for the modulation were: displacement of the Lyot stop by around $10 \%$ of the projected diameter of the main mirror $(D)$, rotation by 1 degree at a time, and change in size from 90 to $75 \%$ of $D$. Figure 1 shows schematically portions of the field transmitted by the Lyot stop vs. time. 25 wavefronts are propagated for each position of the Lyot stop. This number is determined by the atmospheric coherence time, taken to be around $40 \mathrm{~ms}$ at $2.2 \mu \mathrm{m}$, and the exposure time per image which is set to 1sec. Fourier transform of the field after the Lyot stop gives the electric field in the detector plane, and the squared modulus of this Fourier transform yields the final on-axis PSF. For the images of planets the electric field corresponding to AO-residuals and instrumental aberrations is propagated only through the Lyot stop, giving the off-axis PSF. We scale and shift this 

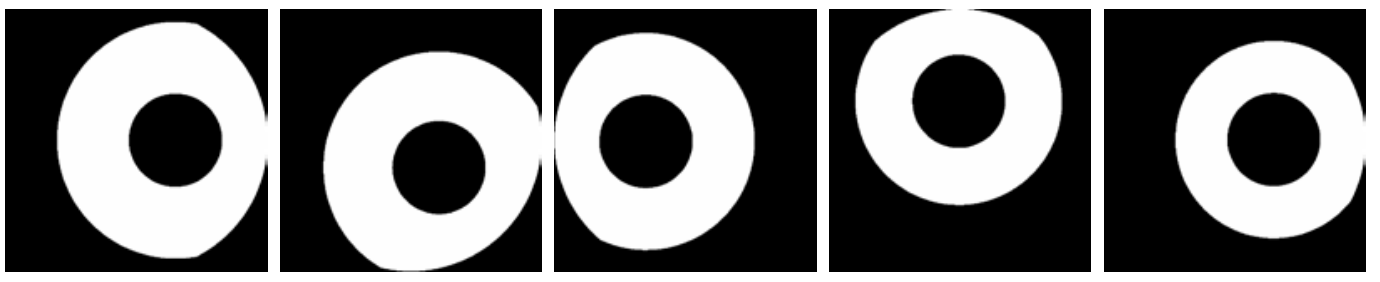

Fig. 1. Schematic illustrations of the pupil shape upon introduction of the modulated Lyot stop for the angles of 0, 45, 180, 270 and 360 degrees. The eccentric masking results in the PSF which is elongated in one direction, while the shrinking of the mask yields PSFs with Airy rings and speckles growing in size.

off-axis PSF to simulate seven planets located at various separations from the star, within the range of $0.1-0.35$ arcsec. The contrast ratios are between $10^{5}$ and $10^{6}$. The 25 "instantaneous" images are co-added to produce one long exposure, and we generate 360 such exposures, i.e. one cycle of the Lyot-stop modulation. Finally, Poisson, background $\left(14 \mathrm{mag} / \operatorname{arcsec}^{2}\right)$ and readout noise $\left(10 \mathrm{e}^{-}\right)$are added to each image.

\section{Results}

Figure 2 illustrates how we can achieve deep nulls at the locations of the static speckles. The same speckles remain at the constant intensity level throughout the Lyot simulation. At the locations of the planets the Lyot stop modulation translates to a periodic intensity fluctuation which is offset from zero by the constant flux from a planet. We tried several approaches to de-modulate the images, i.e. remove this intensity fluctuation:

- Fitting of sinusoids or splines superimposed on a sloping background; this is dictated by the fact that peak of the planetary PSF is linearly decreasing throughout one cycle. This decrease is the result of less light coming through the Lyot stop, as well as the overall growth of the Airy pattern, which translates to less light per pixel. The fitting was not successful because it called for too many degrees of freedom and eventually "over-fitted" the modulation, removing the planetary signal.

- Spectral deconvolution [18]; this technique was originally proposed for imaging spectroscopy with an Integral Field Spectrograph (IFS). The method exploits linear scaling of the wavefronts with wavelength which results in linear scaling of the PSFs: the Airy pattern expands, while the planet if present - stays fixed. The IFS data cube is then re-scaled so that the Airy patterns are re-aligned and the planetary PSF traces a line through the cube. The next step is the estimation of the stellar PSF for each wavelength, which can be done by fitting of the low-order polynomial to each spaxel. The stellar contribution is then subtracted from the data cube, and the residuals are scaled back to the original coordinates so that the planetary signal falls on the same pixel again. The method was only partly successful in dealing with our modulated PSFs, even when only the size of the Lyot stop was changing. This is because the ratio of diameters of the masked central obscuration and the outer edge of the Lyot stop was changing, which resulted in non-trivial spatial scaling of the PSFs.

- Displaying of the minimum intensity for each pixel proved to be most successful. This simplified variant of the "dark speckle" algorithm relies only on the fact that at a location of a possible companion intensity would never reach zero (noise notwithstanding).

In Figure 3 we show the minimum for each pixel in a modulated data cube vs. a long-exposure image produced by co-adding all 360 images from the "static" Lyot simulation. The difference is very 

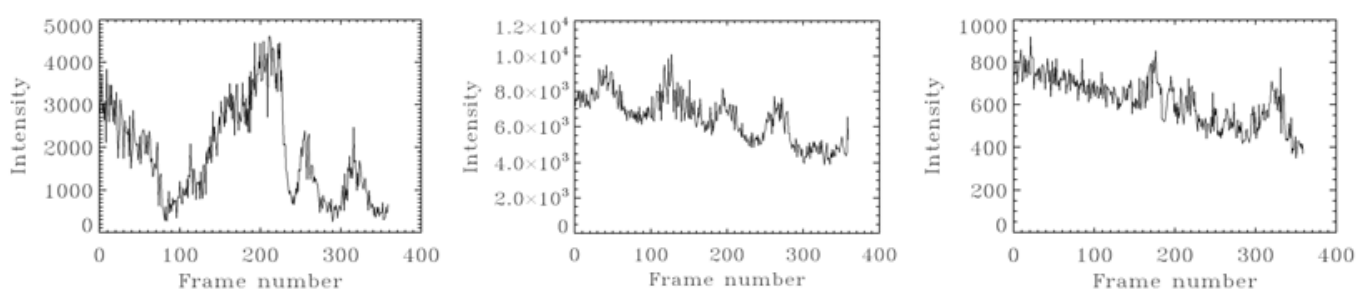

Fig. 2. Left: time series of intensity for a static speckle (location a in Figure 3). Centre: planet close to the star (location b in Figure 3). Right: planet further away from the star (location $\mathbf{c}$ in Figure 3).
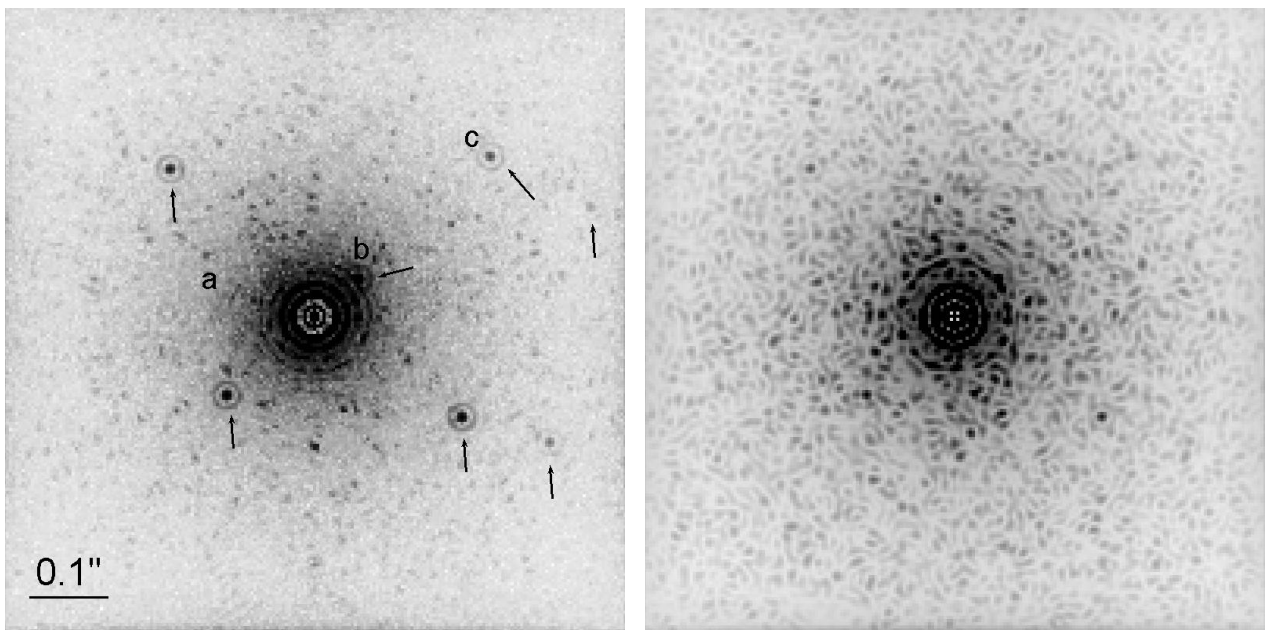

Fig. 3. Left: minimum for each pixel in a modulated data cube; arrows denote the locations of the seven planets. Right: long exposure from the simulation of the classic Lyot setup. Both images are displayed on the square-root scale; the central parts are deliberately saturated to bring out the faint speckle structure outside the PSF core. Colors are inverted for better contrast.

clear: all seven planets are visually detectable in the first image, while hardly 2 planets can be reliably detected in the second one. This is because for the duration of the Lyot simulation static speckles keep landing on the same pixels, and are therefore indistinguishable from real companions. Of course, in reality static aberrations are not truly static, but rather quasi-static and they will slowly average-out over time. In this paper we showed how this process can be accelerated.

\section{Conclusions}

By using a detailed simulation of the high-contrast imaging system on the E-ELT we demonstrated how aperture modulation could be employed to shift the Airy pattern and randomize the persistent speckles. The device can be implemented in the laboratory using an off-axis diaphragm. It remains to be tested how efficient this scheme is in polychromatic light. We also intend to try new post-processing schemes. The disadvantage of "dark speckle" is that it returns the minimum value, which will be less than the true flux from the planet because of the Strehl ratio variations [19], as well as Poisson noise.

\section{References}

1. Beuzit, J.-L. et al., The Messenger 125, (2006) 29 
2. Macintosh, B. et al., SPIE Proceedings 7015, (2008) 701518

3. Lyot, B., Monthly Notices of the Royal Astronomical Society 99, (1939) 580

4. Soummer, R., Astrophysical Journal 618, (2005) L161

5. Martinez, P., Boccaletti, A., Kasper, M., Cavarroc, C., Yaitskova, N., Fusco, T., Verinaud, C., Astronomy and Astrophysics 492, (2008) 289

6. Aime, C., Soummer, R., Astrophysical Journal 612, (2004) L85

7. Soummer, R., Ferrari, A., Aime, C., Jolissaint, L., Astrophysical Journal 669, (2007) 642

8. Marois, C., Lafreniere, D., Macintosh, B., Doyon, R., Astrophysical Journal 673, (2008) 647

9. Racine, R., Walker, G., Nadeau, D., Doyon, R., Marois, C., Publications of the Astronomical Society of the Pacific 111, (1999) 587

10. Gisler, D. et al., SPIE Proceedings 5492, (2004) 463

11. Labeyrie, A., Astronomy and Astrophysics 298, (1995) 544

12. Ribak, E., Gladysz, S., Optics Express 16, (2008) 15553

13. Kasper, M. et al., SPIE Proceedings 7015, (2008) 70151S

14. Jolissaint, L., Veran, J.-P., Conan, R., Journal of the Optical Society of America A 23, (2006) 382

15. http://www.eso.org/sci/facilities/eelt/science/drm/tech_data/ao

16. McGlamery, B. L., in Image Processing, J. C. Urbach, ed., SPIE Proceedings 74, (1976) 225

17. Cavarroc, C., Boccaletti, A., Baudoz, P., Fusco, T., Rouan, D., Astronomy and Astrophysics 447, (2006) 397

18. Sparks, W., Ford, H., Astrophysical Journal 578, (2002) 543

19. Gladysz, S., Christou, J., Bradford, L. W., Roberts, L. C., Publications of the Astronomical Society of the Pacific 120, (2008) 1132 